major volcanic eruptions (or the lack of such eruptions) were the main factor behind cold (or warm) swings that persisted for several decades. Shifts in greenhouse-gas concentrations had a smaller, but still detectable, imprint. The team found no indication that variations in the Sun's radiation output affected mean global temperature over the same time frames.

In general, physics-based climate models accurately reproduce proxy estimates of our climate's history over the past millennium. However, these models exaggerate the degree of cooling caused by the two largest volcanic eruptions of the Common Era: the AD 1257 Samalas and the AD 1815 Tambora eruptions in Indonesia $^{11}$. This discrepancy implies that we cannot be sure how bitter a chill would follow a similar eruption in the future.

The familiar maxim that the climate is always changing is certainly true. But even when we push our perspective back to the earliest days of the Roman Empire, we cannot discern any event that is remotely equivalent either in degree or extent - to the warming over the past few decades. Today's climate stands apart in its torrid global synchrony.

Scott St. George is in the Department of Geography, Environment and Society, University of Minnesota, Minneapolis, Minnesota 55455, USA.

e-mail:stgeorge@umn.edu
1. Matthews, J. A. \& Briffa, K. R. Geogr. Ann. A 87, 17-36 (2005).

2. Mann, M. E. et al. Science 326, 1256-1260 (2009).

3. Neukom, R., Steiger, N., Gómez-Navarro, J. J., Wang, J. \& Werner, J. P. Nature 571, 550-554 (2019).

4. PAGES 2 k Consortium. Nature Geosci. https://doi. org/10.1038/s41561-019-0400-0 (2019).

5. Esper, J. et al. Dendrochronologia 50, 81-90 (2018).

6. Tierney, J. E. et al. Paleoceanography 30, 226-252 (2015).

7. PAGES2k Consortium. Sci. Data 4, 170088 (2017).

8. Cook, E. R., Briffa, K. R., Meko, D. M., Graybill, D. A. \& Funkhouser, G. Holocene 5, 229-237 (1995).

9. McGregor, H. V. et al. Nature Geosci. 8, 671-677 (2015).

10. Huybers, K., Rupper, S. \& Roe, G. H. Clim. Dyn. 46, 3709-3723 (2016).

11.Sigl, M. et al. Nature 523, 543-549 (2015).

\title{
Cell communication in the blink of an eye
}

The bodies of unicellular organisms called protists can contract extremely fast. Analysis reveals that the flow of surrounding fluid during contraction triggers a chain reaction of contraction of neighbouring protists. SEE LETTER P.560

\section{PAVEL TOMANCAK}

$\mathrm{P}$ assing information reliably and rapidly across large distances is crucial for the survival of cells in complex environments. Multicellular organisms have evolved ways to pass signals along neurons at speeds of 100 metres per second. In the unicellular world, organisms rely on their external medium when transmitting signals between cells. Mathijssen et al. ${ }^{1}$ report on page 560 that when unicellular organisms called protists undergo rapid cellular contraction, the fluid in which the organisms live is stirred up, and the resulting fluid flow can trigger the ultra-fast propagation of contraction behaviour across the protist population. Contraction can be accompanied by the release of toxins as a form of defence ${ }^{2}$. The ability to trigger a population-level wave of contractions might be crucial for survival of protists in a perilous aquatic environment full of predators.

The hero of our story is a protist called Spirostomum ambiguum (Fig. 1). Although small by our standards, its length of $1.3 \mathrm{~mm}$ makes it a giant among unicellular organisms. Ever since the biologist Ernst Haeckel reported classic studies of protists in the late nineteenth century ${ }^{3}, S$. ambiguum has been a topic of fascination, particularly because it can contract to about $40 \%$ of its original length at a speed 100 times faster than the blink of an eye $e^{4,5}$. Although biologists have come a long way towards understanding the cellular and molecular mechanisms underlying this contraction $^{6-8}$, it has remained a relatively neglected subject in biological research.

Now Mathijssen and colleagues have explored this topic from a biophysical viewpoint, and their results remind us of how incredibly fast this process is. Using high-speed video microscopy that can capture 10,000 frames per second, the authors filmed the contractions of thousands of S. ambiguum cells, and quantified their contraction speeds comprehensively.

They find that, during its 5-millisecond contraction, the protist accelerates to reach the equivalent of a gravitational force ( $g$ force) of $14 \mathrm{~g}$. This is highly impressive, considering that pilots in the Master Class group of the Red Bull Air Race (a Formula One racing equivalent for aeroplanes) are disqualified if they exceed $12 g$, because a pilot who experiences such forces is at risk of losing consciousness. Yet such $g$ forces pose no problems for S. ambiguum. After the rapid contraction, the protist relaxes comparatively slowly, within about 1 second, and can repeat the cycle again and again.

After quantifying the key parameters of the contraction, Mathijssen and colleagues studied

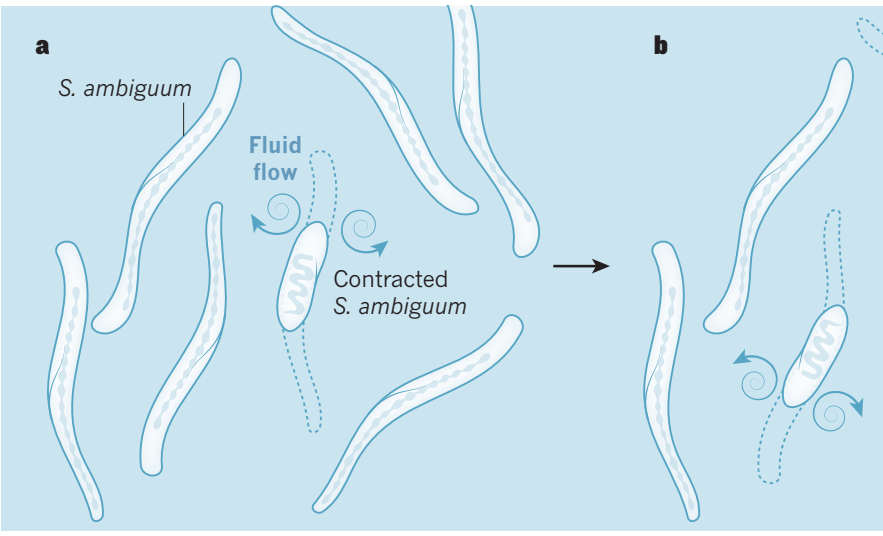

Figure 1 | The spread of a wave of contraction across a protist population. a, The protist Spirostomum ambiguum lives in aquatic environments and has the capacity to contract to less than half its normal length ${ }^{4,5}$. Mathijssen et al. ${ }^{1}$ studied this phenomenon, and report that when this

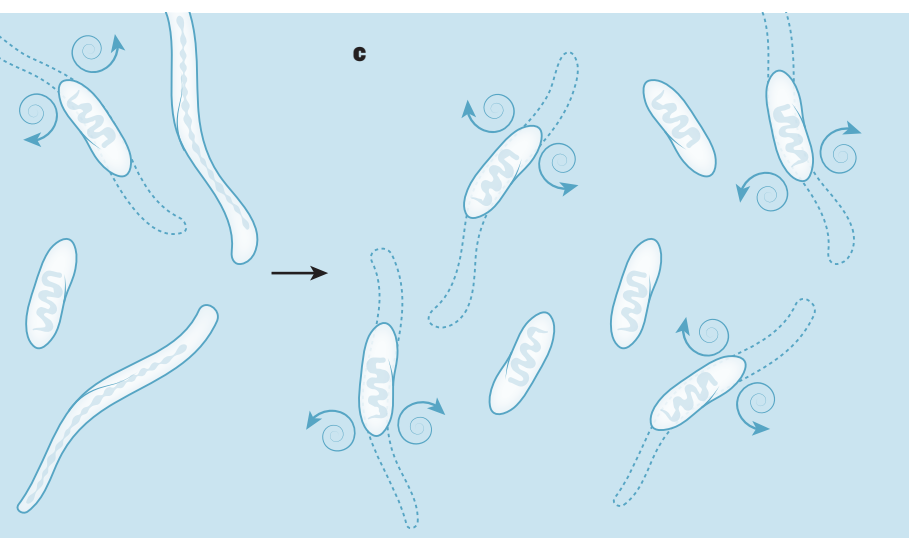

five-millisecond contraction occurs, it generates flows in the surrounding fluid. b. If these flows are sensed by neighbouring S. ambiguum cells, this causes them to contract. c, The contraction can thus rapidly spread through the cell population. 
the phenomenon in more depth as they sought to understand its effects in the microscopic world and determine the implications. The authors observed that the contraction generates long-range fluid flows around the organism. When they used beads to visualize the flows, they found that they look like vortices that expand over time. Using well-established equations that describe fluid motion ${ }^{9}$, the authors recapitulated this flow pattern in a computer simulation. This showed that, in a sufficiently liquid medium, the contraction could generate flow that facilitated the dispersal of material around the protist.

But what triggers the contraction? Biologists had thought of this behaviour as a type of 'startle' response, perhaps a reaction to the presence of a predator. However, the exact mechanism had remained elusive. Mathijssen and colleagues propose that the trigger for contraction could be flows in the surrounding fluid generated by the organisms themselves, or by predators.

To test their hypothesis, they constructed an apparatus in which individual S. ambiguum cells were exposed to fluid flows at increasing velocities. Using fluid-dynamics methods ${ }^{10}$, the authors could relate the velocity of the flow that triggers a contraction to the level of tension that such flows induce in biological membranes. They find a remarkable similarity between the level of flow-induced tension that triggers contraction and the tension required to open mechanosensitive ion channels in cell membranes ${ }^{11}$. It has been suggested that protists use mechanosensitive channels to sense liquid flows ${ }^{12}$, and this convergence of theory and experimental work suggests that, once flows reach a certain threshold of magnitude, a contraction is triggered.

At this point, however, the happy union of biology and physics ends. This is because, to satisfy a biologist that such a mechanism is at play, evidence would be required that depletion of these mechanosensitive channels in S. ambiguum cells affects the organisms' ability to react to the liquid flow. Such manipulations are not currently feasible in S. ambiguum, and so, for now, we must regard this mechanism as not definitively proven.

Regardless of the exact molecular details of how cells contract, Mathijssen and colleagues' experiments have clearly established that flows can trigger the contraction. Given their observation that the contraction itself generates flows, the authors then asked what happens when many $S$. ambiguum cells come together in close proximity. Can they trigger each other's contractions by means of the flows they generate? Remarkably, they can. S. ambiguum cells cultured in vitro tend to self-assemble into clusters, and the authors report that, when the cells reach a certain density, the protists exhibit a striking collective phenomenon: one cell spontaneously contracts, triggering contractions in its neighbours and thereby propagating a wave of

contraction through the colony (Fig. 1).

These waves travel at remarkably high speeds, of $0.25 \mathrm{~m} \mathrm{~s}^{-1}$. The authors used further powerful theoretical frameworks ${ }^{13-15}$ to simulate the triggering and propagation of the waves, generating different simulations depending on the orientation, shape and density of the S. ambiguum cells. They compared these theoretical analyses with their experimental results, and established the threshold cellular density at which S. ambiguum colonies are likely to generate fast collective contractions.

But the million-dollar question remains: what is this collective contraction useful for? The authors show, through simulations, that one possible function

"Contraction generates long-range fluid flows around the organism." of waves of contraction is to enable a synchronized discharge of toxins in response to a predator. The flows generated by a large predator itself, or by a predator's attempt to eat an individual S. ambiguum ${ }^{16}$, might generate an initial trigger that induces widespread toxin release by the colony into the surrounding medium. Testing this hypothesis would require targeted genetic manipulations to uncouple the flow-generation and -sensing process from the toxin-release event.

Many protists exhibit rapid contractions, and many cells and organisms, such as bacteria and fishes, generate and sense liquid flows. Investigating the generation and sensing of these flows is likely to be a fruitful topic for future research. lt will be interesting to see how interdisciplinary cooperation between biology and physics continues to shed light on this remarkable signal-propagation process.

Pavel Tomancak is at the Max Planck Institute of Molecular Cell Biology and Genetics,

Dresden 01307, Germany.

e-mail:tomancak@mpi-cbg.de

1. Mathijssen, A. J. T. M., Culver, J., Bhamla, M. S. \& Prakash, M. Nature 571, 560-564 (2019).

2. Buonanno, F., Guella, G., Strim, C. \& Ortenzi, C. Hydrobiologia 684, 97-107 (2012).

3. Haeckel, E. Das protistenreich. Eine populäre uebersicht über das formengebiet der niedersten lebewesen (Günther, 1878); https://doi. org/10.5962/bhl.title.58542

4. Jones, A. R., Jahn, T. L. \& Fonseca, J. R. J. Cell. Physiol. 68, 127-133 (1966).

5. Hawkes, R. B. \& Holberton, D. V. J. Cell. Physiol. 84 225-235 (1974).

6. Lehman, W. J. \& Rebhun, L. I. Protoplasma 72 153-178 (1971).

7. Ishida, H. \& Shigenaka, Y. Cytoskeleton 9, 278-282 (1988).

8. Yagiu, R. \& Shigenaka, Y. J. Protozool. 10, 364-369 (1963).

9. Winkler, R. G. Eur. Phys. J. Spec. Top. 225, 2079-2097 (2016)

10.Cortez, R., Fauci, L. \& Medovikov, A. Phys. Fluids 17, 031504 (2005)

11.Phillips, R., Kondev, J., Theriot, J. \& Garcia, H. G. Physical Biology of the Cell (Garland, 2012).

12. Ohmura, T. et al. Proc. Natl Acad. Sci. USA 115, 3231-3236 (2018).

13.Xia, W. \& Thorpe, M. F. Phys. Rev. A 38, 2650-2656 (1988).

14. Takeuchi, K. A., Kuroda, M., Chaté, H. \& Sano, M. Phys. Rev. Lett. 99, 234503 (2007).

15. Balanis, C. A. Proc. IEEE 80, 7-23 (1992).

16. Buonanno, F. Biologia 66, 648-653 (2011).

\title{
OCEAN SCIENCE
}

\section{Marine heatwaves in a changing climate}

\begin{abstract}
Heatwaves in the ocean can rapidly disrupt marine ecosystems and the economies that depend on them. A global analysis of these events casts light on their causes and sets the stage for revealing how they might change in the future.
\end{abstract}

\section{MICHAEL G. JACOX}

$\mathrm{H}$ eatwaves that occur over land are well known for having adverse impacts on human health, infrastructure and agriculture. Less attention has been paid to analogous episodes in the ocean, dubbed marine heatwaves (MHWs), but interest in these transient events is growing as their potentially dramatic ecological and economic impacts ${ }^{1}$ have become clear. This enhanced awareness of the importance of MHWs has fostered a desire to understand their causes and whether they can be predicted. Writing in Nature
Communications, Holbrook et al. ${ }^{2}$ present the first comprehensive analysis of MHWs across the globe. They identify specific drivers of these events, as well as associations between MHWs and known climate oscillations.

On a local scale, MHWs can be induced by anomalous ocean heating at the ocean surface (caused by changes in air temperature, winds or cloud cover, for example), or as a result of horizontal or vertical currents and mixing in the surrounding ocean. These local MHWdriving processes are often tied to large-scale climate oscillations, and Holbrook et al. have mapped out such relationships globally for 